ROM2F/2009/02, NSF-KITP-09-13

\title{
From Twists and Shifts to L-R asymmetric D-branes ${ }^{1}$
}

\author{
Massimo Bianchi \\ Dipartimento di Fisica and Sezione I.N.F.N. \\ Università di Roma "Tor Vergata" \\ Via della Ricerca Scientifica, 00133 Roma, Italy \\ and \\ Kavli Institute for Theoretical Physics \\ University of California, Santa Barbara, CA 93106-4030
}

\begin{abstract}
In the first part of the talk, we discuss non-geometric twists and shifts and briefly review asymmetric orbifolds and free fermion constructions. These allow us to build Type IIB models with $\mathcal{N}=1_{L}+1_{R}$ and $\mathcal{N}=1_{L}+0_{R}$ models having few or no moduli. We then consider unoriented projections of the former and ('exotic') D-branes in the latter.

In the second part, devoted to L-R asymmetric D-branes, we review how extended supergravity vacua in $D=4$ can be embedded in Type II superstrings. We then identify bound states of D-branes with residual susy and non-trivial R-R couplings. We discuss the $\mathcal{N}=6=2_{L}+4_{R}$ case in detail and sketch other extended susy cases. Finally we describe the resulting open string excitations.
\end{abstract}

We conclude with some speculations and possible interesting developments.

\footnotetext{
${ }^{1}$ Talk delivered at the 4-th RTN "Forces-Universe" EU network Workshop in Varna, September 2009.
} 


\section{Foreword}

So far, the combined effect of twists and shifts has not been systematically explored in the context of (unoriented) strings [1]. The basic idea is that chiral twists can freeze out untwisted moduli, while (orthogonal) non-geometric shifts prevent massless twisted moduli from appearing.

In this way, one may provide exact CFT descriptions of 'T-folds' [2] in terms of (a)symmetric orbifolds [3, 4] and/or free fermions [5, 6] and allow for a systematic search of perturbative vacua with few moduli. Moreover they suggest the existence of 'new' kinds of L-R asymmetric D-branes [7] with largely unexplored phenomenological applications.

In the era of LHC, taming superstring vacua with few or no moduli is more than necessary $[8,9]$.

The talk is divided into two parts.

In the first part, based on [10], we will discuss twists and shifts, briefly review asymmetric orbifolds $[3,4]$ and free fermions $[5,6]$, build Type IIB models with $\mathcal{N}=1_{L}+1_{R}$ and

$\mathcal{N}=1_{L}+0_{R}$ from $T_{S O(12)}^{6}$, find consistent unoriented projections and ('exotic') D-branes [11]

In Part II, devoted to L-R asymmetric D-branes and based on [7], we will discuss extended supergravity vacua in string theory [12], identify bound states of D-branes with residual susy and non-trivial $R-R$ couplings, focus on the $\mathcal{N}=6=2_{L}+4_{R}$ case, sketch other extended susy cases, and describe the resulting open string excitations.

We will end with an outlook.

\section{2 (Unoriented) T-folds with few or no T's}

\subsection{Asymmetric orbifolds and free fermions}

Asymmetric orbifolds [3, 4] are constructions where Left- and Right-moving fields on the string worldsheet are treated differently or are different altogether. Stringent modular invariance constraints must be satisfied.

For simplicity, consider $Z_{2 L}$ chiral reflections of Left-moving internal bosonic and fermionic coordinates

$$
\mathcal{I}_{i}: \quad X_{L}^{i} \rightarrow-X_{L}^{i}, \quad X_{R}^{i} \rightarrow X_{R}^{i}, \quad \psi^{i} \rightarrow-\psi^{i}, \quad \tilde{\psi}^{i} \rightarrow \tilde{\psi}^{i}
$$

Similarly one can act on the Right-movings. 
In a $Z_{2 L} \times Z_{2 L}^{\prime} \times Z_{2 R} \times Z_{2 R}^{\prime}$ orbifold with generators $\mathcal{I}_{3456}, \mathcal{I}_{1256}$ and $\overline{\mathcal{I}}_{3456}, \overline{\mathcal{I}}_{1256}$ all untwisted moduli fields, except the axio-dilaton are frozen [10].

If one combines chiral twists with chiral shifts along orthogonal directions such as

$$
\sigma_{j}: X_{L}^{j} \rightarrow X_{L}^{j}+\delta^{j}, \quad X_{R}^{j} \rightarrow X_{R}^{j} \quad ;
$$

with $2 \delta$ a lattice vector, most massless twisted moduli are prevented from appearing. Clearly such duality twists and shifts are symmetries of very special tori, in particular those based on free fermions $[5,6,12]$. In these constructions one 'fermionizes' the internal coordinates, so that the worldsheet supercurrent becomes [13]

$$
G=\Psi^{\mu} \partial X_{\mu}+\psi^{i} y^{i} w^{i}
$$

Preservation of $G$, up to a sign, under parallel transport along non-contractible cycles requires that the boundary conditions of the various fermions be related. For 'real' fermions, i.e. $Z_{2}$ twists, one has b.c. $\left(\Psi^{\mu}\right)=$ b.c. $\left(\psi^{i}\right)+$ b.c. $\left(y^{i}\right)+$ b.c. $\left(w^{i}\right), \forall i$ and $\mu$.

The construction is specified by the choice of basis sets of fermions $b_{\alpha}$. Modular invariance (or level matching) imposes stringent constraints. Focussing on $Z_{2}$ twists one finds [6]

$$
\begin{aligned}
n_{L-R}\left(b_{\alpha}\right) & =0 \bmod 8 ; \\
n_{L-R}\left(b_{\alpha} \cap b_{\beta}\right) & =0 \bmod 4 ; \\
n_{L-R}\left(b_{\alpha} \cap b_{\beta} \cap b_{\gamma}\right) & =0 \bmod 2 ; \\
n_{L-R}\left(b_{\alpha} \cap b_{\beta} \cap b_{\gamma} \cap b_{\delta}\right) & =0 \bmod 2 ;
\end{aligned}
$$

where $n_{L-R}(b)=n_{L}(B)-n_{R}(b)$ denotes the difference between the numbers of L- and Rmoving fermions in set $b$. Notice that combinations such as $y^{i} w^{i}$ in any basis set act as non-geometric shifts.

In what follows, our starting point will be the Type IIB superstring on the $T^{6}$ maximal torus of $S O(12)$ with $\mathcal{N}=8=4_{L}+4_{R}$. The presence of a non vanishing B-field plays a subtle crucial role in the reduction of the number of twisted sectors [14]. For our purposes, the fermionic description of the model relies on the choice of

$$
F=\left\{\psi^{1 \ldots 8} y^{1 \ldots 6} w^{1 \ldots 6} \mid \tilde{\psi}^{1 \ldots 8} \tilde{y}^{1 \ldots 6} \tilde{w}^{1 \ldots 6}\right\}, \quad S=\left\{\psi^{1 \ldots 8}\right\}, \quad \tilde{S}=\left\{\tilde{\psi}^{1 \ldots 8}\right\}
$$

as basis sets. The one-loop 'torus' partition function reads

$$
\mathcal{T}_{(4,4)}=\left|V_{8}-S_{8}\right|^{2}\left(\left|O_{12}\right|^{2}+\left|V_{12}\right|^{2}+\left|S_{12}\right|^{2}+\left|C_{12}\right|^{2}\right)
$$

where $O, V, S, C$ denote characters characters of $S O(2 n)$ current algebra [15] that can be expressed in terms of Jacobi $\vartheta$ functions. 
Alternatively, keeping only $F$ and $S$, one finds an $\mathcal{N}=4=4_{L}+0_{R}$ model with $S U(2)^{6}$ gauge group and $S O(20)$ 'pseudo-symmetry' [16]. Its partition function reads

$$
\mathcal{T}_{(4,0)}=\left(V_{8}-S_{8}\right)\left(O_{12} \bar{V}_{20}+V_{12} \bar{O}_{20}-S_{12} \bar{S}_{20}-C_{12} \bar{C}_{20}\right)
$$

There has been recent revival of interest on free fermion constructions both for heterotic strings, where spinor/vector duality has been 'established' and realistic models proposed, as well as for Type II strings where (non) magic hyper-free supergravities have been constructed and models with few (twisted) moduli found [14, 17].

Our analysis, aimed at finding unoriented T-folds with few or no T's, was largely motivated by two seemingly unrelated investigations the CDMP model [18] and DJK 'minimal' model [19].

The CDMP model, based on a previous observation in [20], is a standard geometric freely acting orbifold $T^{6} / Z_{2} \times Z_{2}$, that yields Type I / Heterotic dual pairs. All twisted moduli are massive. Only untwisted moduli $T_{I}, U_{I}$ survive. Including gaugino condensate(s) in the open string sector and/or 3-form fluxes [21] allows to partially stabilize the dilaton and other moduli.

The DJK 'minimal' model [19], a (non magic [22]) hyper-free Type II model with $\mathcal{N}=2=2_{R}+0_{L}$ susy, is a fermionic construction based on the choice of sets: $F, S, \bar{S}, \bar{b}_{1}, b_{1}=\left\{\Psi^{\mu}, \psi^{1,2} ; y^{3,4,5,6}, y^{1} w^{1} \mid \bar{y}^{5} \bar{w}^{5}\right\}, b_{2}=\left\{\Psi^{\mu}, \psi^{3,4} ; y^{1,2}, w^{5,6} y^{3} w^{3} \mid \bar{y}^{6} \bar{w}^{6}\right\}, b_{3}=$ $\left\{\Psi^{\mu}, \psi^{5,6} ; w^{1,2,3,4} y^{6} w^{6} \mid \bar{y}^{6} \bar{w}^{6}\right\}$

Only the dilaton vector (!) multiplet survives. All susy associated to Left-moving supercharges are broken, $\mathcal{N}_{L}=0$, due to the L-R asymmetric $(-)^{F_{L}} \sigma$ freely acting orbifold projection of $T_{S O(12)}^{6}$.

\subsection{The 'minimal' model with " $h_{11} "=" h_{11} "=1$}

In the perspective of unoriented projections, one is lead to replace $\bar{b}_{3}$ with $b_{2}$ and get a L-R symmetric asymmetric orbifold. Geometric (freely acting) projections associated to $b_{1} \bar{b}_{1}$ and $b_{2} \bar{b}_{2}$ are combined with non geometric (freely acting) projections associated to $b_{1}, b_{2}, \ldots b_{1} \bar{b}_{2}$. The surviving spacetime susy is $\mathcal{N}_{L}=\mathcal{N}_{R}=1, \mathcal{N}_{\text {tot }}=2$. All untwisted moduli except the dilaton hypermultiplet are projected out. One could hope that all twisted sectors be massive, thanks to the shifts. Alas, massless multiplets arise from the $b_{1} b_{2} \bar{b}_{1} \bar{b}_{2}$ twisted sector, that contributes one hyper and one vector multiplet. By analogy with 'geometric' CY-like compactifications one is lead to introduce effective Hodge numbers and get " $h_{11} "=" h_{21} "=1$ in this case.

As we will see later, unoriented projections can produce $1_{u}+2_{t}-n$ chiral-plets and $n$ vector-plets $(n=0,1)$. Unfortunately, at a first scan, the open string spectrum seems to 
be non chiral and the Chan-Paton group suffers rank reduction due to the non-vanishing but quantized B-field [23, 24, 25, 26, 27, 28]. A systematic study is under way. MSSM embeddings in similar fermionic constructions have been recently discussed in [30].

Let us now, give some details of the $T^{6} /\left(Z_{2 L} \times Z_{2 L}^{\prime} \times Z_{2 R} \times Z_{2 R}^{\prime}\right)$ model with " $h_{11}$ " = " $h_{21} "=1[10]$.

The generators of the orbifold group can be specified by the following choice of fermionic sets

$$
\begin{aligned}
& b_{1}=\left\{\psi_{3456} ; y_{13456} ; \quad w_{1} \mid \quad ; \quad \tilde{y}_{5} ; \quad \tilde{w}_{5}\right\} \\
& b_{2}=\left\{\psi_{1256} ; \quad y_{123} ; w_{356} \mid \quad ; \quad \tilde{y}_{6} ; \quad \tilde{w}_{6}\right\} \\
& \tilde{b}_{1}=\left\{\quad ; \quad y_{5} ; \quad w_{15} \mid \tilde{\psi}_{3456} ; \tilde{y}_{13456} ; \quad \tilde{w}_{1}\right\} \\
& \tilde{b}_{2}=\left\{\quad ; \quad y_{6} ; \quad w_{6} \mid \tilde{\psi}_{1256} ; \quad \tilde{y}_{123} ; \tilde{w}_{356}\right\}
\end{aligned}
$$

Defining also $b_{3}=b_{1} b_{2}$ and $\tilde{b}_{3}=\tilde{b}_{1} \tilde{b}_{2}$

$$
\begin{aligned}
& b_{3}=\left\{\psi_{1234} ; \quad y_{2456} ; \quad w_{1356} \mid ; \quad \tilde{y}_{56} ; \quad \tilde{w}_{56}\right\} \\
& \tilde{b}_{3}=\left\{\quad ; \quad y_{56} ; \quad w_{56} \mid \tilde{\psi}_{1234} ; \quad \tilde{y}_{2456} ; \quad \tilde{w}_{1356}\right\}
\end{aligned}
$$

Orbifold group elements can then be expressed as $b_{\alpha}^{m} \tilde{b}_{\beta}^{n}$ with $\alpha, \beta=1,2,3, m, n=0,1$.

The torus partition function

$\mathcal{T}=\frac{1}{16}\left\{\sum_{c, d=0}^{3} \rho_{0 c} \bar{\rho}_{0 d} \boldsymbol{\Lambda}_{00, c d}+\sum_{a, b=0}^{3}\left(\rho_{a 0} \bar{\rho}_{b 0} \boldsymbol{\Lambda}_{a b, 00}+\rho_{a a} \bar{\rho}_{b b} \boldsymbol{\Lambda}_{a b, a b}\right)+\sum_{a=1}^{3} \sum_{b \neq a}^{3} \epsilon_{\mathbf{a b}} \rho_{a b} \bar{\rho}_{a b} \boldsymbol{\Lambda}_{a a, b b}\right\}$

where the chiral amplitudes $\rho_{a b}$

$$
\begin{aligned}
\rho_{00}=\frac{1}{\eta^{8}}\left(Q_{o}+Q_{v}\right) \quad \rho_{0 h}=\frac{1}{\eta^{8}}\left(Q_{o}^{(h)}-Q_{v}^{(h)}\right) \\
\rho_{h 0}=\frac{1}{\eta^{8}}\left(Q_{s}^{(h)}+Q_{c}^{(h)}\right) \quad \rho_{h h}=-\frac{i}{\eta^{8}}\left(Q_{s}^{(h)}-Q_{c}^{(h)}\right) \\
\rho_{h h^{\prime}}=-\frac{i}{\eta^{8}}\left(Q_{s-^{\prime}}^{(h)}-Q_{c-^{\prime}}^{(h)}\right) \quad h \neq h^{\prime} \quad h=1,2,3
\end{aligned}
$$

can be expressed in terms of the super-characters

$$
\begin{aligned}
& Q_{o}=V_{4} O_{4}-S_{4} S_{4} \quad, \quad Q_{v}=O_{4} V_{4}-C_{4} C_{4} \\
& Q_{s}=O_{4} S_{4}-C_{4} O_{4} \quad, \quad Q_{c}=V_{4} C_{4}-S_{4} V_{4}
\end{aligned}
$$

while the lattice sums for $h=1,2$ as well as for $h^{\prime}=3$ can be conveniently written in terms of $\vartheta$ functions

$$
\begin{aligned}
& \boldsymbol{\Lambda}_{30,30}=\boldsymbol{\Lambda}_{03,03}^{*}=\frac{i}{2}\left(\vartheta_{4}^{2} \vartheta_{2}^{4} \bar{\vartheta}_{4}^{4} \bar{\vartheta}_{4}^{2}-\vartheta_{4}^{4} \vartheta_{2}^{2} \bar{\vartheta}_{4}^{2} \bar{\vartheta}_{2}^{4}\right) \quad \boldsymbol{\Lambda}_{00, h 0}=\boldsymbol{\Lambda}_{00,0 h}^{*}=\frac{1}{2}\left(\vartheta_{3}^{3} \vartheta_{4}^{3} \bar{\vartheta}_{3}^{5} \bar{\vartheta}_{4}+\vartheta_{3}^{3} \vartheta_{4}^{3} \bar{\vartheta}_{3} \bar{\vartheta}_{4}^{5}\right) \\
& \boldsymbol{\Lambda}_{h 0,00}=\boldsymbol{\Lambda}_{0 h, 00}^{*}=\frac{1}{2}\left(\vartheta_{3}^{3} \vartheta_{2}^{3} \bar{\vartheta}_{3}^{5} \bar{\vartheta}_{2}+\vartheta_{3}^{3} \vartheta_{2}^{3} \bar{\vartheta}_{3} \bar{\vartheta}_{2}^{5}\right) \quad \boldsymbol{\Lambda}_{h 0, h 0}=\boldsymbol{\Lambda}_{0 h, 0 h}^{*}=\frac{i}{2}\left(\vartheta_{4}^{3} \vartheta_{2}^{3} \bar{\vartheta}_{2}^{5} \bar{\vartheta}_{4}-\vartheta_{2}^{3} \vartheta_{4}^{3} \bar{\vartheta}_{2} \bar{\vartheta}_{4}^{5}\right) \\
& \boldsymbol{\Lambda}_{00,30}=\boldsymbol{\Lambda}_{00,03}^{*}=\frac{1}{2}\left(\vartheta_{3}^{2} \vartheta_{4}^{4} \bar{\vartheta}_{3}^{4} \bar{\vartheta}_{4}^{2}+\vartheta_{3}^{4} \vartheta_{4}^{2} \bar{\vartheta}_{3}^{2} \bar{\vartheta}_{4}^{4}\right) \quad \boldsymbol{\Lambda}_{30,00}=\boldsymbol{\Lambda}_{03,00}^{*}=\frac{1}{2}\left(\vartheta_{3}^{2} \vartheta_{2}^{4} \bar{\vartheta}_{3}^{4} \bar{\vartheta}_{4}^{2}+\vartheta_{3}^{4} \vartheta_{2}^{2} \bar{\vartheta}_{3}^{2} \bar{\vartheta}_{2}^{4}\right) \\
& \boldsymbol{\Lambda}_{00,00}=\frac{1}{2}\left(\left|\vartheta_{2}\right|^{12}+\left|\vartheta_{3}\right|^{12}+\left|\vartheta_{4}\right|^{12}\right) \quad \boldsymbol{\Lambda}_{\text {other }}=0
\end{aligned}
$$


As anticipated, the only massless twisted states come from the $b_{3} \tilde{b}_{3}$ sector. The whole massless spectrum is coded in

$$
\mathcal{T}=|V-S-C|^{2}+|2 O-S-C|^{2}+\ldots
$$

and consists in the $\mathcal{N}=2$ supergravity multiplet coupled to 2 hypers and 1 vector, i.e. " $h_{11} "=" h_{21} "=1$.

\subsection{Models with $\mathcal{N}=1_{L}+1_{R}$ based on $T_{S O(12)}^{6}$}

We then performed a systematic search of models with $\mathcal{N}=1_{L}+1_{R}$ from $T_{S O(12)}^{6} / Z_{2}^{4}$, corresponding to the choice of basis sets $F, S, \tilde{S}$ plus four more sets of the form

$$
\begin{aligned}
& b_{1}=I_{3456} \sigma^{i_{1} i_{2} \cdots} \bar{\sigma}^{k_{1} k_{2} \ldots}=\left\{(\psi y)^{3456}(y w)^{i_{1} i_{2} \ldots} \mid(\tilde{y} \tilde{w})^{k_{1} k_{2} \ldots}\right\}, \\
& b_{2}=I_{1256} \sigma^{j_{1} j_{2} \ldots} \bar{\sigma}^{l_{1} l_{2} \ldots}=\left\{(\psi y)^{1256}(y w)^{j_{1} j_{2} \ldots} \mid(\tilde{y} \tilde{w})^{l_{1} l_{2} \ldots}\right\}, \\
& \bar{b}_{1}=\bar{I}_{3456} \sigma^{k_{1} k_{2} \cdots} \bar{\sigma}^{i_{1} i_{2} \ldots}=\left\{(y w)^{k_{1} k_{2} \ldots} \mid(\tilde{\psi} \tilde{y})^{3456}(\tilde{y} \tilde{w})^{i_{1} i_{2} \ldots}\right\}, \\
& \bar{b}_{2}=\bar{I}_{1256} \sigma^{l_{1} l_{2} \ldots} \bar{\sigma}^{j_{1} j_{2} \ldots}=\left\{(y w)^{l_{1} l_{2} \ldots} \mid(\tilde{\psi} \tilde{y})^{1256}(\tilde{y} \tilde{w})^{j_{1} j_{2} \ldots}\right\},
\end{aligned}
$$

As above, in view of the resulting $\mathcal{N}=1_{L}+1_{R}$ supersymmetry, one can introduce "effective" Hodge numbers " $h_{11}$ " $=n_{h}-1$, " $h_{21}$ " $=n_{v}$. We found three finite sequences of models with (" $\left.h_{11} ", " h_{21} "\right)$ :

$$
\begin{aligned}
& (n, n), n=1,2,3,4,5,9, \quad \text { self }- \text { mirror, } \quad \chi=0 \\
& (2 n, 2 n+6) /(2 n+6,2 n), n=0,1,2, \quad \text { mirror pairs, } \quad \chi=\mp 12 \\
& (2 n+3,2 n+15) /(2 n+15,2 n+3), n=0,1, \quad \text { mirror pairs, } \quad \chi=\mp 24
\end{aligned}
$$

Once again, the non vanishing but quantized B-field plays a subtle role in reduction of twisted sectors [14] while discrete torsion [4, 20], i.e. relative signs between disconnected orbits of the modular group in the one-loop partition function, lead to the exchange of vectors and hypers, i.e. generalized mirror symmetry for these non-geometric Type II vacuum configurations. It is amusing to see that $\chi=12 k$ in all cases we analyzed. We have no convincing explanation for this except for the obvious fact that the models at hand can be thought of as non-geometric orbifolds of $K 3 \times T^{2}$.

\subsection{Models with $\mathcal{N}=1_{L}+0_{R}$ based on $T_{S O(12)}^{6}$}

In the same vein, one can systematically scan for models with $\mathcal{N}=1_{L}+0_{R}$ based on $T_{S O(12)}^{6}$. To this end, one keeps only the sets $F$ and $S$ (not $\tilde{S}$ ) plus, for instance, two more sets $b_{1}$ and $b_{2}$. The latter spacetime susy to $\mathcal{N}=1_{L}+0_{R}$ and the internal 
(pseudo)symmetry $S O(20)$. The "true" gauge symmetry can only be determined after a careful analysis of the vertex operators for vectors and their OPE's. Not unexpectedly, it turns out to be a subgroup of $S U(2)^{6}$ with abelian factors and the possibility of further (perturbative) Higgs mechanism.

The massless spectrum can be decomposed according to $\mathcal{T}_{0}=\mathbf{G}_{1}+n_{v} \mathbf{V}_{1}+n_{v^{\prime}} \mathbf{V}_{1}^{\prime}+$ $n_{c} \mathbf{C}_{1}+n_{c^{\prime}} \mathbf{C}_{1}^{\prime}$

$$
\text { with } \begin{aligned}
\mathbf{G}_{1}+\mathbf{C}_{1} & =(V-S-C) \bar{V}, \\
\mathbf{V}_{1} & =(V-S-C) \bar{O} \quad \mathbf{V}_{1}^{\prime}=(S-O) \bar{S}+(C-O) \bar{C}, \\
\mathbf{C}_{1} & =(2 O-S-C) \bar{O} \quad \mathbf{C}_{1}^{\prime}=(S-O) \bar{C}+(C-O) \bar{S},
\end{aligned}
$$

As indicated there are two different kinds of chiral and vector multiplets distinguished by the nature, NS-NS or R-R, of the bosonic components. Though L-R asymmetric, these models allow the introduction of generalized D-branes that couple to (twisted) R-R states $[7]$.

The simplest example we found of a model with $\mathcal{N}=1_{L}+0_{R}$ spacetime susy has $\left(n_{v}, n_{v}^{\prime} ; n_{c}, n_{c}^{\prime}\right)=(14,0 ; 5,0)$ and correspond to the choice of orbifold generators

$$
b_{1}=I_{3456} \sigma_{12} \bar{\sigma}_{45} \quad, \quad b_{2}=I_{1256} \sigma_{36} \bar{\sigma}_{5} .
$$

In the absence of 'exotic' D-branes, the resulting gauge symmetry, associated to worldsheet currents, is $S U(2)^{4} \times U(1)^{2}$. The (pseudo)symmetry is broken according to

$$
O(12)_{L} \times O(20)_{R} \rightarrow\left[O(4)^{2} \times O(2)^{2}\right]_{L} \times\left[O(2)^{2} \times O(16)\right]_{R}
$$

or, rather, $O(16)_{R} \rightarrow O(2) \times O(14)$, with $O(2)$ little group in $D=4$.

We also found other $\mathcal{N}=1_{L}+0_{R}$ Type II models with $\left(n_{v}, n_{v}^{\prime} ; n_{c}, n_{c}^{\prime}\right)=$ $(10,0 ; 25,0),(8,0 ; 27,0),(6,8 ; 13,8),(6,8 ; 29,8)$. As apparent, the scan was neither very systematic nor very inspiring. Yet one should explore the possibility of adding 'exotic' D-branes.

\subsection{Unoriented projections and open strings}

L-R symmetric though non geometric models with $\mathcal{N}=1_{L}+1_{R}$ admit $\Omega$ projections

$$
\operatorname{Tr}_{\mathcal{H}_{L} \otimes \mathcal{H}_{R}} \Omega\left(g^{L} \otimes g^{R}\right)=\operatorname{Tr}_{\mathcal{H}_{L}} g_{\Omega}
$$

$g_{\Omega}$ diagonal action $g^{L} g^{R}$ with Left- and Right- moving fields identified, i.e. $\bar{I}_{i} \rightarrow I_{i}$, $\bar{\sigma}_{i} \rightarrow \sigma_{i}$. In general $g_{\Omega}$ amplitudes are not chiral 'square roots' of amplitudes in torus partition function. Several closed string moduli are odd under $\Omega$ and are thus projected out. D-branes may be needed to cancel R-R tadpoles [1, 15, 31, 32, 33]. 
An unoriented ('Type I') model without open strings can be constructed from the Type II with $\mathcal{N}=1_{L}+1_{R}$ model with ( $h_{11} "=$ " $\left.h_{21} "\right)=(1,1)$. Two allowed Klein-bottle projections are of the form

$$
\mathcal{K}=\frac{1}{16} \sum_{a, b, c, d} \operatorname{Tr}_{\mathcal{H}_{L c} \otimes \mathcal{H}_{R d}} \Omega b_{a} \bar{b}_{b}=\frac{1}{4} \sum_{a, b} \operatorname{Tr}_{\mathcal{H}_{L a}} b_{b \Omega}=\frac{1}{4 \eta^{8}} \sum_{a, b=0}^{3} \epsilon_{a, b} \rho_{a b} \Lambda\left[\begin{array}{l}
a \\
b
\end{array}\right]
$$

with $\Lambda\left[\begin{array}{l}0 \\ 0\end{array}\right]=\vartheta_{3}^{6}+\epsilon \vartheta_{2}^{6}$ and

$$
\begin{array}{lll}
\Lambda\left[\begin{array}{l}
0 \\
h
\end{array}\right]=\vartheta_{4}^{3} \vartheta_{3}^{3}+\epsilon \vartheta_{1}^{3} \vartheta_{2}^{3}, & \Lambda\left[\begin{array}{l}
h \\
0
\end{array}\right]=\vartheta_{2}^{3} \vartheta_{3}^{3}+\epsilon \vartheta_{3}^{3} \vartheta_{2}^{3}, & \Lambda\left[\begin{array}{l}
h \\
h
\end{array}\right]=\vartheta_{1}^{3} \vartheta_{3}^{3}+\epsilon \vartheta_{4}^{3} \vartheta_{2}^{3} \\
\Lambda\left[\begin{array}{l}
0 \\
3
\end{array}\right]=\vartheta_{4}^{2} \vartheta_{3}^{4}+\epsilon \vartheta_{1}^{2} \vartheta_{2}^{4}, & \Lambda\left[\begin{array}{l}
3 \\
0
\end{array}\right]=\vartheta_{2}^{2} \vartheta_{3}^{4}+\epsilon \vartheta_{3}^{2} \vartheta_{2}^{4}, & \Lambda\left[\begin{array}{l}
3 \\
3
\end{array}\right]=\vartheta_{1}^{2} \vartheta_{3}^{4}+\epsilon \vartheta_{4}^{2} \vartheta_{2}^{4}
\end{array}
$$

For $\epsilon=-1, \epsilon_{a, b}=1$, no massless untwisted or twisted tadpoles appear in the transverse channel and thus no D-branes are needed. The resulting Type I model consists of closed strings only with 'minimal' $\mathcal{N}=1$ content $\frac{1}{2}(\mathcal{T}+\mathcal{K})_{\text {massless }}=\mathbf{G}_{1}+2 \mathbf{C}_{1}$

Let us now discuss the simplest unoriented model with open strings. For simplicity, consider $T^{6} / Z_{2 L} \times Z_{2 L}^{\prime} \times Z_{2 R} \times Z_{2 R}^{\prime}$ with no shifts at the $S O(12)$ point. The spectrum can be coded in a basis of 64 super-characters, that appear in the geometric case as well $[15,34]$. There are two choices of signs (discrete torsion), leading to two different Klein bottle projections. Yet, they both lead to the same non-chiral massless open string spectrum, encoded in $(\mathcal{A}+\mathcal{M}) / 2: \mathcal{N}=4 \mathrm{SYM}$ with gauge group $U(N) \times U(4-N)$.

Rank reduction is due to quantized B-field [23, 24, 25, 26, 27, 28]. Alas, there seems to be some tension between chirality and moduli stabilization. Before drawing too drastic conclusions one should wait for a more systematic analysis of D-branes in non geometric compactifications of the above kind.

\section{Bound states of L-R asymmetric D-branes}

\subsection{Type II superstring vacua with extended susy}

Type II superstrings can give rise to (non) geometric vacua with extended supergravity $[12,29]$

$$
\begin{array}{llll}
\mathcal{N}=8 & \leftrightarrow & \mathcal{N}_{L}=4, \mathcal{N}_{R}=4 \\
\mathcal{N}=6 & \leftrightarrow & \mathcal{N}_{L}=2, \mathcal{N}_{R}=4 \\
\mathcal{N}=5 & \leftrightarrow & \mathcal{N}_{L}=1, \mathcal{N}_{R}=4 \\
\mathcal{N}=4 & \leftrightarrow & \mathcal{N}_{L}=2, \mathcal{N}_{R}=2 \quad \text { or } \quad \mathcal{N}_{L}=0, \mathcal{N}_{R}=4 \\
\mathcal{N}=3 & \leftrightarrow & \mathcal{N}_{L}=1, \mathcal{N}_{R}=2 & \\
\mathcal{N}=2 & \leftrightarrow & \mathcal{N}_{L}=1, \mathcal{N}_{R}=1 \quad \text { or } \quad \mathcal{N}_{L}=0, \mathcal{N}_{R}=2
\end{array}
$$


In the spirit of the first part of the talk, asymmetric orbifolds and free fermions, i.e. twists and shifts, can provide 'exact' (rational) CFT descriptions of the above. For our present purpose it is crucial to observe that, even if L-R asymmetric, whenever massless $\mathrm{R}$-R states (e.g. graviphotons) survive there must be bound-states of D-branes they couple to $[31,32]$. As we will see [7], these 'exotic' D-branes preserve some fraction of extended susy and satisfy BPS conditions. In many cases, one can resort to CFT techniques, i.e. use boundary states for magnetized D-branes [35, 37, 40, 36, 39, 38], to determine the resulting open string excitations.

\section{$3.2 \mathcal{N}=6=2_{L}+4_{R}$ case}

Spontaneous breaking $\mathcal{N}=8 \rightarrow \mathcal{N}=6$ via chiral $Z_{2}$ twist of the L-movers corresponds to T-duality twists on four internal directions, $T_{t}^{4}$ )

$$
X_{L}^{i} \rightarrow-X_{L}^{i} \quad, \quad \Psi_{L}^{i} \rightarrow-\Psi_{L}^{i} \quad, \quad i=6,7,8,9
$$

accompanied by an order two shift along the untwisted $T_{s}^{2}[12]$. Unbroken susy's satisfy $\mathcal{Q}_{L}=\Gamma_{6789} \mathcal{Q}_{L}$, while no conditions are to be imposed on $\mathcal{Q}_{R}$. After dualizing all masseless 2-forms into axions, the $30=2_{N S-N S}+12_{N S-N S}+16_{R-R}$ scalar moduli parameterize the coset space $\mathcal{M}_{\mathcal{N}=6}^{D=4}=S O^{*}(12) / U(6)$. The $16=8_{N S-N S}+8_{R-R}$ vectors together with their magnetic duals transform according to the $\mathbf{3 2}$ dimensional chiral spinor representation of $S O^{*}(12)$.

Let us now consider $\mathcal{N}=6$ BPS conditions for D-brane bound-states invariant under twist and shift that couple to the surviving R-R graviphotons and carry the $16_{R-R}=$ $2_{(1 \mid 5)}+4_{(1 \mid 3)}+6_{(3 \mid 3)}+4_{(5 \mid 3)}$ R-R charges

$$
q_{1}^{a}+\frac{1}{4 !} \varepsilon_{i j k l} q_{5}^{a i j k l} \quad, \quad q_{1}^{i}+\frac{1}{3 !} \varepsilon^{i}{ }_{j k l} q_{3}^{j k l} \quad, \quad q_{3}^{a i j}+\frac{1}{2 !} \varepsilon^{i j}{ }_{k l} q_{3}^{a k l} \quad, \quad q_{5}^{a b i j k}+\varepsilon^{i j k}{ }_{l} q_{3}^{a b l}
$$

Consider for instance the state consisting of a D5 wrapped along the twisted $T_{t}^{4} \times S_{s}^{1}$ and a D1 along the same $S_{s}^{1}$. The susy condition, $\mathcal{Q}_{R}=\Gamma_{04} \Gamma_{6789} \mathcal{Q}_{L}=\Gamma_{04} \mathcal{Q}_{L}$, leads to a $1 / 3$ BPS state. A different analysis applies to BPS states carrying NS-NS charges e.g. the two massive gravitini and their superpartners, carrying internal generalized KK momentum, form a complex 1/2 BPS multiplet.

D-branes in 'T-folds' have already been studied from different vantage points [41, 11 , $44,43,46,45]$ but never in a systematic way.

Other $\mathcal{N}=6$ cases can be studied. First, instead of $Z_{2}$ one can perform a $Z_{n}$ chiral projection on 4 real (2 complex) super-coordinates as

$$
\left(Z^{1}, Z^{2}\right)_{L} \rightarrow\left(\omega Z^{1}, \omega^{-1} Z^{2}\right)_{L} \quad, \quad\left(\Psi^{1}, \Psi^{2}\right)_{L} \rightarrow\left(\omega \Psi^{1}, \omega^{-1} \Psi^{2}\right)_{L}
$$


with $\omega^{n}=1$. In order to avoid massless twisted states, one has to combine it with an order $n$ shift along the 'untwisted' directions $\left(Z_{L}^{3} ; Z_{R}^{i}\right)$. Alternatively, the maximal torus of $S U(3)^{3}$ admits a chiral $Z_{3}$ projection with no shift. $\mathcal{N}=5$ supergravity survives in the untwisted sector. The twisted sector produces an extra massless gravitino multiplet that completes the spectrum of $\mathcal{N}=6$ supergravity [29].

\subsection{Other extended susy cases with $\mathbf{L} \neq \mathbf{R}$}

Let us list the other extended susy cases with some of their properties

- $\mathcal{N}=5=1_{L}+4_{R}$, unique massless spectrum, non-geometric, uncorrected LEEA (like $\mathcal{N}=6,8)$

- $\mathcal{N}=4=2_{L}+2_{R}$ uncorrected LEEA, variable $N_{v}$, (non)geometric, $S L(2) \times S O\left(6, N_{v}\right)$ symmetry

- $\mathcal{N}=3=1_{L}+2_{R}$ uncorrected LEEA, variable $N_{v}$, non-geometric / fuxes, $U\left(3, N_{v}\right)$ symmetry

- $\mathcal{N}=2=1_{L}+1_{R}$, (non) geometric, quantum corrections absent in special cases $(\chi=0$, eg FHSV, octonionic magic [22])

- $\mathcal{N}=4=0_{L}+4_{R}, \mathcal{N}=2=0_{L}+2_{R}, \mathcal{N}=1=0_{L}+1_{R}$ NO massless R-R graviphotons, yet massless $R-R$ vectors couple to 'exotic' $D$-branes

Let us then sketch the $\mathcal{N}=5,3$ cases.

\section{$3.4 \mathcal{N}=5=1_{L}+4_{R}$ case}

The simple(st) realization [12] is in terms of $Z_{2}^{L} \times Z_{2}^{L}$ projections acting by T-duality along $T_{6789}^{4}$ and $T_{4589}^{4}$ combined with order two shifts. One can then identify $1 / 5$ BPS bound states of D-branes carrying the $8_{R-R}=6_{(1533)}+2_{(3333)}$ surviving R-R charges (invariant orbits)

$$
q_{(1335)}^{I}=q_{1}^{I}+\frac{1}{4 !} \varepsilon_{i_{I} j_{I} k_{I} l_{I}} q_{5}^{I i_{I} j_{I} k_{I} l_{I}}+\frac{1}{3 !} \varepsilon^{I}{ }_{J, K^{\prime} L^{\prime}} q_{3}^{J K^{\prime} L^{\prime}}+\frac{1}{3 !} \varepsilon^{I}{ }_{J, K^{\prime \prime} L^{\prime \prime}} q_{3}^{J K^{\prime \prime} L^{\prime \prime}}
$$

where $i_{I}, j_{I}, k_{I}, l_{I}$ run over the four directions orthogonal to $T_{I}^{2}$ while $K^{\prime}, L^{\prime}$ and $K$ ", $L$ " run over the two sets of two directions orthogonal to $T_{I}^{2}$ and

$$
q_{(3333)}^{I_{1} I_{2} I_{3}}=q_{3}^{I_{1} I_{2} I_{3}}+\frac{1}{2 !} \varepsilon^{I_{2} I_{3}}{ }_{J_{2} J_{3}} q_{3}^{I_{1} J_{2} J_{3}}+\frac{1}{2 !} \varepsilon^{I_{3} I_{1}}{ }_{J_{3} J_{1}} q_{3}^{J 1 I_{2} J_{3}}+\frac{1}{2 !} \varepsilon^{I_{1} I_{2}}{ }_{J_{1} J_{2}} q_{3}^{J_{1} J_{2} I_{3}}
$$


Other realizations of $\mathcal{N}=5=1_{L}+4_{R}$ are possible, all lead invariably to the unique $\mathcal{N}=5$ supergravity massless spectrum and LEEA: graviton $g_{\mu \nu}$ and 5 gravitini $\psi_{\mu}$, 10 graviphotons $A_{\mu}, 11$ dilatini $\chi$ and 10 scalars $\phi$. The latter parameterize $\mathcal{M}_{\mathcal{N}=5}^{D=4}=$ $S U(5,1) / U(5) .10_{e}+10_{m}$ graviphotons in 20 of $S U(5,1)$ (3-index anti-symmetric tensor). The "Minimal" $\mathcal{N}=5$ superstring solutions have been classified into four classes [12]. Two alternative superstring constructions [29] are available. A $Z_{7}$ asymmetric orbifold of the $S U(7)$ torus $\theta_{L}=\left(\omega_{7}, \omega_{7}^{2}, \omega_{7}^{4}\right)$ and $7 \sigma_{R}=(1,2,-3,0,0,0,0)$ and a $Z_{3}$ asymmetric orbifold of the $S U(3)^{3}$ torus $\theta_{L}=\left(\omega_{3}, \omega_{3}, \omega_{3}\right)$ and $3 \sigma_{R}=(1,-1,0 ; 1,-1,0 ; 1,-1,0)$.

\section{5 $\mathcal{N}=3=1_{L}+2_{R}$ case}

The simplest $\mathcal{N}=3$ model with 3 matter vector-plets can be constructed in two steps. First one performs a 'geometric' $Z_{2}$ freely acting orbifold (locally equivalent to $K 3 \times T^{2}$ ). The $Z_{2}$ action combines a twist breaking $\mathcal{N}=8=4_{L}+4_{R}$ to $\mathcal{N}=4=2_{L}+2_{R}$ and a shift preventing massless twisted states. Then a non geometric chiral (say Left-) projection combined with a shift along the orthogonal directions $a=4,5$ breaks half of the $\mathcal{Q}_{L}$. The surviving NS-NS charges are $p_{R}^{a}$ and their magnetic duals $\hat{P}_{R}^{a}$. The surviving R-R charges are the T-duality invariant combinations

$$
q_{1}^{a}+\frac{1}{3 !} \varepsilon_{b i j}^{a} q_{3}^{b i j} \quad, \quad q_{3}^{a i j}+\frac{1}{3 !} \varepsilon_{b k l}^{a} q_{5}^{b i j k l}
$$

At most bound-states of the above can be 1/3 BPS states. No 1/2 BPS states are allowed in $\mathcal{N}=3$ supergravity. In particular the massive gravitino in $\mathcal{N}=4 \rightarrow \mathcal{N}=3$ belong to a long multiplet.

More $\mathcal{N}=3=1_{L}+2_{R}$ cases and otherwise can be found. A complete classification of "minimal" $Z_{2} \times Z_{2}^{L} \mathcal{N}=3$ superstring solutions [12] lead to four classes with $3+4 K$ matter vector multiplets, with $K=0,1,2$, and eleven sub-classes. Adding an extra chiral projection (ie splitting geometric $Z_{2}$ into two chiral $Z_{2}$ ) produces models with $1+2 K$ matter vector multiplets, with $K=0,1,2$. In particular a model can be found with only one vector multiplet, thus having only three complex scalar moduli (including dilaton!). Another construction is based on the asymmetric $Z_{3}$ projection with $\theta=$ $\left(\omega_{3}, \omega_{3}, \omega_{3} ; 1, \omega_{3}, \omega_{3}^{-1}\right)$ acting on the lattice of $S U(3)^{3}$.

Due to the rigidity of the LEEA and thus the scalar geometry $U\left(3, N_{v}\right) / U(3) \times U\left(N_{v}\right)$ one expects duality with $\mathcal{N}=3$ unoriented D3-brane models with 3-form fluxes [47].

\subsection{Boundary states for L-R asymmetric branes}

In order to identify the open string excitations of the 'exotic' D-branes whose existence we have argued for above, one can resort to the boundary state formalism $[35,37,40,36$, 
$39,38]$.

The boundary state can be factorized into a bosonic part and a fermionic part. For the bosonic coordinates one has

$$
\left|B_{a}\right\rangle^{(X)}=\sqrt{\operatorname{det}\left(\mathcal{G}_{a}+\mathcal{F}_{a}\right)} \exp \left(-\sum_{n>o} a_{-n}^{i} R_{i j}\left(F_{a}\right) \tilde{a}_{-n}^{j}\right)\left|0_{a}\right\rangle
$$

where $R_{a}=\left(1-F_{a}\right) /\left(1+F_{a}\right)$ and $\left|0_{a}\right\rangle \leftrightarrow p_{L}=-R_{a} p_{R}$ For the fermions in the NS-NS sector (where no fermionic zero-modes are present) one finds

$$
\left|B_{a}, \pm\right\rangle_{N S-N S}^{(\psi)}=\exp \left( \pm i \sum_{n \geq 1 / 2} \psi_{-n}^{i} R_{i j}\left(F_{a}\right) \tilde{\psi}_{-n}^{j}\right)| \pm\rangle
$$

while in the R-R sector one has

$$
\begin{gathered}
\left|B_{a}, \pm\right\rangle_{R-R}^{(\psi)}=\frac{1}{\sqrt{\operatorname{det}\left(\mathcal{G}_{a}+\mathcal{F}_{a}\right)}} \exp \left(i \pm \sum_{n>0} \psi_{-n}^{i} R_{i j}\left(F_{a}\right) \tilde{\psi}_{-n}^{j}\right) \mathcal{U}_{A \tilde{B}}^{ \pm}\left(F_{a}\right)|A, \tilde{B}\rangle \\
\mathcal{U}_{A \tilde{B}}^{ \pm}\left(F_{a}\right)=\left[\operatorname{AExp}\left(-F_{i j}^{a} \Gamma^{i j} / 2\right) C \Gamma_{11} \frac{1 \pm i \Gamma_{11}}{1 \pm i}\right]_{A \tilde{B}} .
\end{gathered}
$$

One can compute the partition functions (direct loop channel) and determine the closed string couplings in the transverse tree channel, where the amplitude is simply given by the overlap of the boundary states. With these techniques magnetized and/or intersecting D-branes in L-R symmetric orbifolds have been described [21]. Our aim here is to generalize to $Z_{N_{L}}^{L} \times Z_{N_{R}}^{R}$ action, and construct invariant boundary states of the form

$$
|B, F\rangle_{g}=\frac{1}{\sqrt{N_{L} N_{R}}}\left(1+g_{L}+g_{R}+\ldots+g_{L}^{N_{L}-1} g_{R}^{N_{R}-1}\right)|B, F\rangle=\frac{1}{\sqrt{N_{L} N_{R}}} \sum_{l, r}\left|B, F_{(l, r)}\right\rangle
$$

where the 'induced' magnetic field $F_{(l, r)}$ is determined by the condition $R\left(F_{(l, r)}\right)=$ $R\left(g_{L}^{l}\right) R(F) R^{t}\left(g_{R}^{r}\right)$.

The annulus amplitude then reads

$$
\mathcal{A}_{g, h}=\Lambda(g, h) \mathcal{I}(g, h) \sum_{\alpha} c_{\alpha}^{G S O} \frac{\vartheta_{\alpha}(0)}{\eta^{3}} \prod_{I} \frac{\vartheta_{\alpha}\left(\epsilon_{I}(g, h) \tau\right)}{\vartheta_{1}(\epsilon(g, h) \tau)}
$$

where $g=g_{L} g_{R}, h=h_{L} h_{R}, \Lambda(g, h)$ is the lattice invariant under $g h=g_{L} h_{L} g_{R} h_{R}$, while $\mathcal{I}(g, h)$ is the 'intersection' number counting the invariant discrete zero-modes and finally $\epsilon_{I}(g, h)$ are related to the eigenvalues of $g h=g_{L} h_{L} g_{R} h_{R} \rightarrow \operatorname{diag}\left(e^{2 i \epsilon_{I}(g, h)}\right)$.

As an example consider the $\mathcal{N}=5$ model based on the $T^{6} / Z_{3}^{L}$ torus of $S U(3)^{3}$. Prior to twists and shifts, there are 27 boundary states, associated to the 'integrable' representations of the current algebra at level $\kappa=1, \mathcal{A}_{\vec{r}, \vec{s}}=N_{\vec{r}, \vec{s}}^{\vec{t}} \mathcal{X}_{\vec{t}}$, where $\mathcal{X}_{\vec{t}}=\left(V_{8}-S_{8}\right) \chi_{t_{1}} \chi_{t_{2}} \chi_{t_{3}}$ correspond to D-branes with magnetic quantum number $(n, m)=(1,0),(-1,1),(0,-1)$. 
After twist and shift, invariant states consist of branes rotated and displaced wrt one another

$$
\mathcal{A}_{Z_{3}^{L \neq R}}=\frac{1}{6} \sum_{a, b \in Z_{3}} \Lambda_{(a, b)} \mathcal{I}_{(a, b)} \sum_{\alpha} c_{\alpha}^{G S O} \frac{\vartheta_{\alpha}(0)}{\eta^{3}} \prod_{I} \frac{\vartheta_{\alpha}(a \tau+b)}{\vartheta_{1}(a \tau+b)}
$$

As a result both 'untwisted' and 'twisted' strings are present [11, 48], leading to a non chiral spectrum in this case.

\section{Outlook}

Let us summarize what we said and speculate on possible interesting developments of our analyses.

We hope we have convinced the audience, that twists and shifts produce (unoriented) models with few or no T's that can incorporate other mechanisms of moduli stabilization, e.g. open [49, 50, 51, 37, 52] and closed string fluxes [21], (non) anomalous $U(1)$ 's [53, 54], instanton effects, ... . Explicit computations are feasible, though very systematic scans may be very time-consuming (thousands of characters, ...) [55]. We have found a L-R symmetric 'minimal' model with only $1+1$ twisted moduli [10] that escaped previous scans $[17,30]$ but $\ldots$ a perturbative L-R symmetric Type II model with " $h_{11} "=" h_{21} "=0$ is yet to be found! Discrete 'deformations' (B-field etc) play a subtle role and can widen phenomenological perspectives and, hopefully, reduce some 'tension' we observed between chirality and moduli stabilization

D-branes in L-R asymmetric vacua, e.g. $\mathcal{N}=1_{L}+0_{R}$, offer new possibilities [7]. The relation with (non) geometric fluxes is yet to be understood and interacting CFT's (WZW, Gepner or alike) to be explored. In particular, abstract SCFT with one-loop (super) characters $\mathcal{X}_{0}$ (identity), $\mathcal{X}_{i}$ (massless chiral), $\mathcal{X}_{i}^{c}$ (massless anti-chiral), $\mathcal{X}_{I}$ (massive $h_{I}>$ 1/2) may admit 'exotic' modular invariants of the form

$$
\mathcal{T}_{I I B}=\left|\mathcal{X}_{0}\right|^{2}+\sum_{i}\left[\mathcal{X}_{i} \overline{\mathcal{X}}_{I(i)}+\mathcal{X}_{i}^{c} \overline{\mathcal{X}}_{I(i)}^{c}+\overline{\mathcal{X}}_{i} \mathcal{X}_{I(i)}+\overline{\mathcal{X}}_{i}^{c} \mathcal{X}_{I(i)}^{c}\right]+\ldots
$$

where $I(i)$ labels massive characters with $h_{I(i)}=1 / 2+n_{I}$ (if present). All moduli except the dilaton (multiplet) would be 'stabilized'. One cannot do any better for perturbative strings in Minkowski space. So far, we have not been able to find a L-R symmetric model of this kind but we will endure since we are unaware of any no-go theorem and the 'minimal' DJK model [19], though L-R asymmetric, offers good hopes.

Alternatively, one can start from Type I / Heterotic on $T^{4} / Z_{2}[15,53]$. No neutral twisted moduli are present since they are eaten by anomalous $U(1)$ 's), only untwisted ones. Compactifying on $T^{2}$ and projecting by a freely acting $Z_{2}$ can eliminate (some) untwisted 
moduli and produce no extra twisted moduli. Including unoriented D-brane instantons [54] and open string fluxes, one can hope to find a 'phenomenologically' viable model with all moduli stabilized by mechanisms that are under full control of CFT techniques on the worldsheet.

\section{Acknowledgments}

I would like to thank P. Anastasopoulos, J. F. Morales Morera and G. Pradisi for a very enjoyable collaboration on some of the topics covered in my talk. I would to thank the organizers of the 4-th RTN "Forces-Universe" EU network Workshop in Varna, and M. Chichmanova for the very kind hospitality. I delivered similar talks at Mathematical Challenges in String Phenomenology (ESI, Vienna, October 2008) and at Fundamental Aspects of Superstring Theory (KITP, Santa Barbara, January 2009), where this work has been completed. I would like to thank the organizers for creating a stimulating environment. This work was supported in part by MIUR-PRIN contract 2007-5ATT78, NATO PST.CLG.978785, RTN grants MRTNCT- 2004-503369, EU MRTN-CT-2004512194, MRTN-CT-2004-005104 and in part by the National Science Foundation under Grant No. PHY05-51164.

\section{References}

[1] A. Sagnotti, arXiv:hep-th/0208020. G. Pradisi and A. Sagnotti, Phys. Lett. B 216, 59 (1989). M. Bianchi and A. Sagnotti, Phys. Lett. B 247 (1990) 517. M. Bianchi and A. Sagnotti, Nucl. Phys. B 361 (1991) 519. For a review, see for instance C. Angelantonj and A. Sagnotti, Phys. Rept. 371 (2002) 1 [Erratum-ibid. 376 (2003) 339] [arXiv:hep-th/0204089].

[2] M. Dine and E. Silverstein, arXiv:hep-th/9712166.

[3] K. S. Narain, M. H. Sarmadi and C. Vafa, Nucl. Phys. B 288 (1987) 551.

[4] C. Vafa, Nucl. Phys. B 273 (1986) 592.

[5] H. Kawai, D. C. Lewellen and S. H. H. Tye, Phys. Rev. Lett. 57 (1986) 1832 [Erratumibid. 58 (1987) 429], Phys. Rev. D 34 (1986) 3794, Nucl. Phys. B 288 (1987) 1.

[6] I. Antoniadis, C. P. Bachas and C. Kounnas, Nucl. Phys. B 289 (1987) 87. I. Antoniadis and C. Bachas, Nucl. Phys. B 298 (1988) 586.

[7] M. Bianchi, Nucl. Phys. B 805 (2008) 168 [arXiv:0805.3276 [hep-th]]. 
[8] D. Lust, S. Stieberger and T. R. Taylor, Nucl. Phys. B 808 (2009) 1 [arXiv:0807.3333 [hep-th]].

[9] I. Antoniadis, arXiv:0710.4267 [hep-th];

[10] P. Anastasopoulos, M. Bianchi, J. F. Morales and G. Pradisi, arXiv:0901.0113 [hepth].

[11] M. Bianchi, J. F. Morales and G. Pradisi, Nucl. Phys. B 573 (2000) 314 [arXiv:hepth/9910228].

[12] S. Ferrara and C. Kounnas, Nucl. Phys. B 328 (1989) 406.

[13] P. Di Vecchia, V. G. Knizhnik, J. L. Petersen and P. Rossi, Nucl. Phys. B 253, 701 (1985). I. Antoniadis, C. Bachas, C. Kounnas and P. Windey, Phys. Lett. B 171 (1986) 51.

[14] A. E. Faraggi, C. Kounnas, S. E. M. Nooij and J. Rizos, Nucl. Phys. B 695, 41 (2004) [arXiv:hep-th/0403058]. A. E. Faraggi, S. Forste and C. Timirgaziu, JHEP 0608 (2006) 057 [arXiv:hep-th/0605117]. A. E. Faraggi, C. Kounnas and J. Rizos, Phys. Lett. B 648, 84 (2007) [arXiv:hep-th/0606144]. A. E. Faraggi, E. Manno and C. Timirgaziu, Eur. Phys. J. C 50 (2007) 701 [arXiv:hep-th/0610118].

[15] M. Bianchi, Ph. D Thesis, University of Rome "Tor Vergata", 1992; ROM2F-92-13

[16] L. J. Dixon, V. Kaplunovsky and C. Vafa, Nucl. Phys. B 294 (1987) 43.

[17] R. Donagi and K. Wendland, arXiv:0809.0330 [hep-th].

[18] P. G. Camara, E. Dudas, T. Maillard and G. Pradisi, Nucl. Phys. B 795 (2008) 453 [arXiv:0710.3080 [hep-th]].

[19] Y. Dolivet, B. Julia and C. Kounnas, JHEP 0802 (2008) 097 [arXiv:0712.2867 [hepth]].

[20] C. Vafa and E. Witten, J. Geom. Phys. 15 (1995) 189 [arXiv:hep-th/9409188].

[21] For reviews, see e.g. M. Grana, Phys. Rept. 423 (2006) 91 [arXiv:hep-th/0509003]; R. Blumenhagen, B. Kors, D. Lust and S. Stieberger, Phys. Rept. 445 (2007) 1 [arXiv:hep-th/0610327], and references therein.

[22] M. Bianchi and S. Ferrara, JHEP 0802, 054 (2008) [arXiv:0712.2976 [hep-th]].

[23] M. Bianchi, G. Pradisi and A. Sagnotti, Nucl. Phys. B 376 (1992) 365.

[24] M. Bianchi, Nucl. Phys. B 528, 73 (1998) [arXiv:hep-th/9711201]. 
[25] E. Witten, JHEP 9802, 006 (1998) [arXiv:hep-th/9712028].

[26] C. Angelantonj and R. Blumenhagen, Phys. Lett. B 473 (2000) 86 [arXiv:hepth/9911190].

[27] C. Bachas, M. Bianchi, R. Blumenhagen, D. Lust and T. Weigand, JHEP 0808, 016 (2008) [arXiv:0805.3696 [hep-th]].

[28] I. Pesando, Phys. Lett. B 668 (2008) 324 [arXiv:0804.3931 [hep-th]].

[29] A. Dabholkar and J. A. Harvey, JHEP 9902, 006 (1999) [arXiv:hep-th/9809122].

[30] E. Kiritsis, M. Lennek and B. Schellekens, arXiv:0811.0515 [hep-th].

[31] J. Dai, R. G. Leigh and J. Polchinski, Mod. Phys. Lett. A 4, 2073 (1989).

[32] J. Polchinski, Phys. Rev. Lett. 75, 4724 (1995) [arXiv:hep-th/9510017].

[33] E. G. Gimon and J. Polchinski, Phys. Rev. D 54, 1667 (1996) [arXiv:hep-th/9601038].

[34] M. Berkooz and R. G. Leigh, Nucl. Phys. B 483 (1997) 187 [arXiv:hep-th/9605049].

[35] A. Abouelsaood, C. G. . Callan, C. R. Nappi and S. A. Yost, Nucl. Phys. B 280, 599 (1987).

[36] C. G. . Callan, C. Lovelace, C. R. Nappi and S. A. Yost, Nucl. Phys. B 293, 83 (1987).

[37] M. Bianchi and E. Trevigne, JHEP 0601, 092 (2006) [arXiv:hep-th/0506080].

[38] P. Di Vecchia, A. Liccardo, R. Marotta, F. Pezzella and I. Pesando, arXiv:hepth/0601067.

[39] P. Di Vecchia, M. Frau, I. Pesando, S. Sciuto, A. Lerda and R. Russo, Nucl. Phys. B 507, 259 (1997) [arXiv:hep-th/9707068].

[40] M. Billo, P. Di Vecchia, M. Frau, A. Lerda, I. Pesando, R. Russo and S. Sciuto, "Microscopic string analysis of the D0-D8 brane system and dual R-R Nucl. Phys. B 526, 199 (1998) [arXiv:hep-th/9802088].

[41] I. Brunner, A. Rajaraman and M. Rozali, Nucl. Phys. B 558, 205 (1999) [arXiv:hepth/9905024].

[42] C. Angelantonj, R. Blumenhagen and M. R. Gaberdiel, "Asymmetric orientifolds, brane supersymmetry breaking and non-BPS Nucl. Phys. B 589, 545 (2000) [arXiv:hep-th/0006033]. 
[43] M. Gutperle, JHEP 0008, 036 (2000) [arXiv:hep-th/0007126].

[44] M. R. Gaberdiel and S. Schafer-Nameki, Nucl. Phys. B 654, 177 (2003) [arXiv:hepth/0210137].

[45] A. Lawrence, M. B. Schulz and B. Wecht, JHEP 0607, 038 (2006) [arXiv:hepth/0602025].

[46] S. Kawai and Y. Sugawara, JHEP 0802, 027 (2008) [arXiv:0709.0257 [hep-th]].

[47] A. R. Frey and J. Polchinski, Phys. Rev. D 65, 126009 (2002) [arXiv:hep-th/0201029].

[48] R. Blumenhagen, L. Goerlich, B. Kors and D. Lust, "Noncommutative compactifications of type I strings on tori with magnetic JHEP 0010, 006 (2000) [arXiv:hepth/0007024].

[49] I. Antoniadis and T. Maillard, Nucl. Phys. B 716 (2005) 3 [arXiv:hep-th/0412008].

[50] E. Dudas and C. Timirgaziu, Nucl. Phys. B 716 (2005) 65 [arXiv:hep-th/0502085].

[51] M. Bianchi and E. Trevigne, JHEP 0508 (2005) 034 [arXiv:hep-th/0502147].

[52] I. Antoniadis, A. Kumar and T. Maillard, arXiv:hep-th/0505260. Nucl. Phys. B 767, 139 (2007) [arXiv:hep-th/0610246].

[53] M. Berkooz, R. G. Leigh, J. Polchinski, J. H. Schwarz, N. Seiberg and E. Witten, Nucl. Phys. B 475, 115 (1996) [arXiv:hep-th/9605184].

[54] M. Bianchi and J. F. Morales, JHEP 0802, 073 (2008) [arXiv:0712.1895 [hep-th]].

[55] T. P. T. Dijkstra, L. R. Huiszoon and A. N. Schellekens, Nucl. Phys. B 710, 3 (2005) [arXiv:hep-th/0411129]. 